\title{
Traffic Light Controller System using Optical Flow Estimation
}

\author{
Neha Parashar \\ Department of Computer Science and Engineeirng \\ Mewar University \\ Chittorgarh, India
}

\author{
Shiv Kumar \\ Department of Computer Science and Engineeirng \\ Mewar University \\ Chittorgarh, India
}

\begin{abstract}
As we seen everyday vehicle traffic increases day by day on road is causing many issues. We face many traffic jams due to the inefficient traffic controlling system which is unable to cope up with the current scenario of traffic in our country. To overcome such drastic scenario and looking at current traffic volume we need to develop a system which works on real time processing and works after determining the traffic density and then calculating the best possibility in which the traffic on particular cross road is dissolved. Also, it helps in saving time as on traffic roads. In present traffic control system when there is no traffic on road but the static signal not allow traffic to move to cross and it changes after at fixed interval so at every cycle this amount of time is wasted for unused traffic density road and if one road is at high traffic it continuously grows till human intervention. The basic theme is to control the traffic using static cameras fixed on right side of the road along top of the traffic pole to check the complete traffic density on other side of the road. This system will calculate number of vehicles on the road by moving detection and tracking system developed based on optical flow estimation and green light counter will be based on the calculated number of vehicles on the road.
\end{abstract}

Keywords: Optical flow estimation, Moving object detection, tracking, Morphological operation, Blob analysis, Camera.

\section{INTRODUCTION}

In the current scenario of fixed time traffic lights, many a times situation arises wherein there is heavy incoming traffic only from one side of the intersection and the rest are relatively empty. In this case the people on the heavily occupied side have to wait for unreasonably long time as the green light timer is fixed for each side which fails to take in to account that there is no traffic to pass from the other sides. This prolonged waiting time increases the average waiting time of every person in the traffic. Though care is taken while setting these timers by government officials according to the proportionate amount of traffic present on different sides of the intersection but this can never be so flexible as to adapt to the dynamic traffic throughout the day. Moreover, some areas of high traffic volume may receive scanty traffic at some point of the day and some low traffic volume areas might get congested likewise, which leads to an additional increase in waiting time because these timers are set according to average volume of traffic corresponding to the different areas of the city respectively but not according to the different hours of the day. This fixed nature of the present traffic light timers turns out to be ineffective not only during the day but also during late night hours. At night, when there is negligible traffic present, the timers lead to an unreasonably long waiting time. Additionally most people, seeing empty roads ahead of them, tend to jump lights at this point, which quite often lead to accidents [1].

Our proposed system takes into account all these issues by dynamically changing traffic light timers. It intelligently recognizes the volume of the traffic at each side of the intersection thereby providing an adequate amount of time for the traffic to pass. The system monitors traffic throughout the day and takes care of the inability of the fixed timers to adjust 
as per the traffic. Since our system regulates the flow of traffic at night also, it helps in minimizing the chances of an accident.

\section{LITERATURE SURVEY}

E. Atkociunas, R. Blake, A. Juozapavicius, M. Kazimianec (2005) presented paper on "Image processing in road traffic analysis". In this Researches and developments have been performed in image a processing technique which is applied to traffic data collection and analysis [2].

Pejman Niksaz (2012) has proposed a paper "Automatic traffic estimation using image Processing" that estimates the size of traffic in highways by using image processing has been proposed and as a result a message is shown to inform the number of cars in highway [3].

P.D. Kamble, S.P. Untawale and S.B. Sahare (2012) presented paper on "Application of image processing for traffic queue length" to measure queue parameters accurately, traffic queue algorithm is used [4].

Raad Ahmed Hadi, Ghazali Sulong and Loay Edwar George (2014) presented paper on "Vehicle Detection and Tracking Techniques: A Concise Review" in which they present a concise overview of image processing methods and analysis tools which used in building these previous mentioned applications that involved developing traffic surveillance systems [5].

Ramesh Navi, Aruna M. G. (2014) presented paper on "Traffic Event Detection using Computer Vision" they proposed a system in which process includes the subtasks of data collection, negative positive separation, creating training samples, creating description files, haar training and a strong action program which can detect vehicles and the traffic events [6].

Pallavi Choudekar, Sayanti Banerjee, Prof. M.K.Muju presented paper on "Real Time Traffic Control using Image Processing" propose a system for controlling the traffic light by image processing. The system will detect vehicles through images instead of using electronic sensors embedded in the pavement [7].

Overall, the references have advantages such as reporting of speed violation, traffic congestion, accidents, low cost and setup with good accuracy and speed. Some of the disadvantages occurring are variation of ambient light, 3D images are not

www.ijcat.com supportive, and it is difficult to detect vehicle features in windy and other weather conditions.

\section{PROBLEM STATEMENT}

Most of Traffic Controllers in India are Manual Controlling and Fixed Time Traffic Signalization. They lead to traffic accidents at intersections. These crashes are caused by drivers' frustration because of long intersection delays. Without police or other forms of enforcement, the long delays have led to road crashes caused by drivers' disobedience of traffic signals and dangerous driving maneuvers in an effort to beat the signal so as to avoid the long delay. This is owing to the fact that some drivers will not wait for the green times when there are no vehicles approaching the intersection, thus Red Light Running (RLR) will occur. In addition, drivers will try to change lane so as to be close to the stop line. The fuel emission and environmental aspects have an influence on people living near the intersections.

\section{OBJECTIVE}

1. To minimize estimated vehicle delays and stops.

2. To maximize intersection capacity.

3. To design automate control system for traffic on streets.

\section{PROPOSED SYSTEM}

A scene should be selected from a static camera. Our test movies are selected from urban surveillance videos. Some preprocessing operations have to be done for making the scene ready to process. After that propose algorithm apply on video show below:

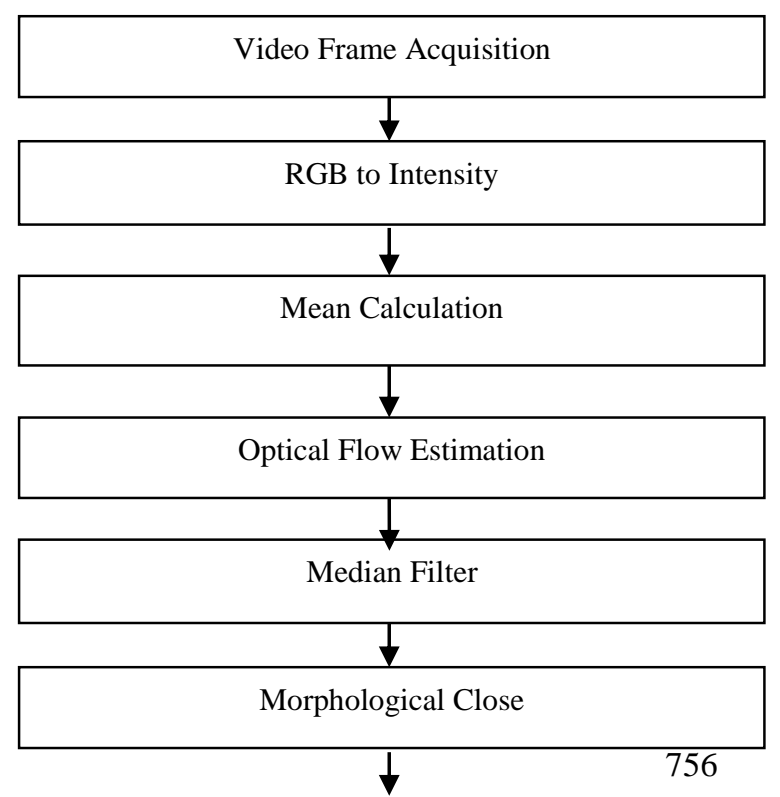




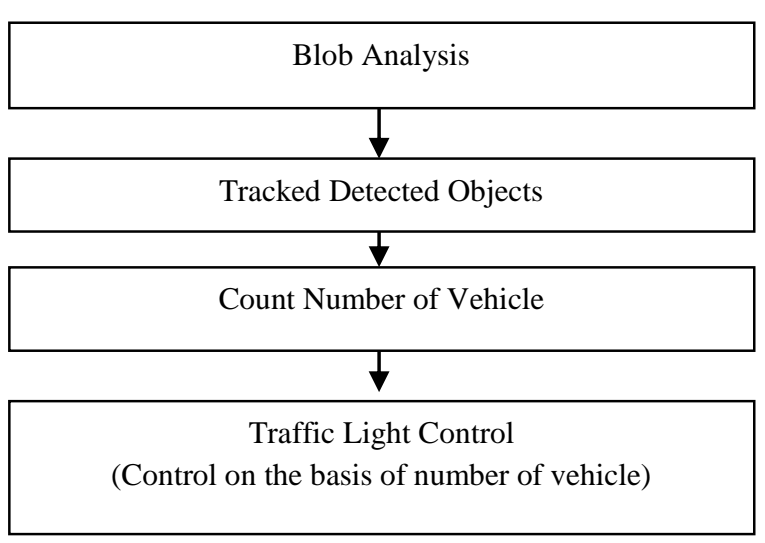

Rules for green light signaling \& time control:

Rule 1: If

Number of vehicle $<0$

Then green light counter $=5 \mathrm{sec}$

Rule 2: If

Number of vehicle $<15$

Then green light counter $=10 \mathrm{sec}$

Rule 3: If

Number of vehicle $<35$

Then green light counter $=30 \mathrm{sec}$

Else

green light counter $=60 \mathrm{sec}$

In proposed system we set maximum time counter to $60 \mathrm{sec}$ and minimum time counter to $5 \mathrm{sec}$. Here we show three type of case. We decide the green light counter according to distance and time. In how much time a particular distance travel by vehicle. The counter time depends on distance because the distance at every intersection road is different.

\section{THEORETICAL WORK}

To design advance traffic control system firstly we setup a camera at 11 feet height. Record video from the camera and perform multiple operations on it discussed below:

\subsection{RGB to Intensity}

First step for this project is to convert the video we intend to simulate from RGB to intensity. RGB (red, green, blue) are the three colors that can be mixed to become any other colors in the color spectrum. In an RGB video, each pixel is represented by a combination of these colors. Though it www.ijcat.com provides a more accurate visual representation of the recorded object(s), having to detect 3 colors in every pixel is redundant. Hence, the simplest way is to convert the video from RGB to intensity. What converting the video to intensity does is represent each pixel in the video with a value ranging from 0 to 255 . 0 being the color black; 255 being the color white. Any values in-between are shades of gray.

\subsection{Mean Calculation}

The mean of every frame is calculated on gray-scale format.

\subsection{Optical Flow Estimation}

Optical flow has been used in conventional video surveillance systems to detect motion, but the purpose of using optical flow in such systems is just to detect moving objects. However, the purpose of using optical flow in our system is to provide statistical traffic flow information. The role of optical flow in our system is essential and critical to final performance. Thus, application-specific assumptions must be considered to choose an algorithm for optical flow estimation from among the many available optical flow algorithms [10].

The optical flow method tries to calculate the motion between two image frames which are taken at times $t$ and $\mathrm{t}+\delta \mathrm{t}$ at every position. These methods are called differential since they are based on local Taylor Series approximation of the image signal; that is, they use partial derivatives with respect to the spatial and temporal coordinates.

There are two most used methods, namely:

- Lucas-Kanade

- Horn-Schunck

To solve the optical flow constraint equation for $\mathrm{u}$ and $\mathrm{v}$, the Lucas-Kanade method divides the original image into smaller sections and assumes a constant velocity in each section. Then, it performs a weighted least-square fit of the optical flow constraint equation to a constant model for in each section, $\Omega$, by minimizing the following equation:

$$
\sum_{x \in \Omega} W^{2}\left[I_{x} u+I_{y} v+I_{t}\right]^{2}
$$




\subsection{Median Filter}

In signal processing, it is often desirable to be able to perform some kind of noise reduction on an image or signal. The median filter is a nonlinear digital filtering technique, often used to remove noise. Median filtering is very widely used in digital image processing because, under certain conditions, it preserves edges while removing noise [11].

\subsection{Morphological Close}

Morphological operations are performed to extract significant features from images that are useful in the representation and description of the shapes in the region; mostly used in image segmentation and pattern recognition. In the proposed system we used both morphological close and erode, respectively, to remove portions of the road and unwanted objects. After morphological closing operation, on condition that vehicle's appearance is not destroyed, objects including many small holes and separated pixels may be connect into one big actual vehicle shape.

\subsection{Blob Analysis}

In the area of computer vision, blob detection refers to visual modules that are aimed at detecting points and/or regions in the image that differ in properties like brightness or color compared to the surrounding.

\subsection{Vehicle Detecting, Tracking and Counting}

Blob analysis has the functionality to produce many forms of statistics, which is crucial for detecting and tracking. For now, the bounding box option is checked. A bounding box is an M-by-4 matrix of [x y height width] bounding box coordinates, where $\mathrm{M}$ represents the number of blobs and $[\mathrm{x} y]$ represents the upper left corner of the bounding box. As the blob moves, the bounding box will follow. Tracking vehicles with boundary boxes are counted.

\subsection{Calculation for Green Light Timer of Traffic Light Control System}

In this section proposed system provide counter time for green signal light according to the number of the vehicles are present in the video. The counter time for green light decide according to the distance between the intersection and the speed of the vehicle.
In proposed system we manage only green light counter only. System, calculate the number of vehicle present in video. According to the number of vehicle presented in the video set green light timer. The timer for green light varies as number of vehicle varies in detected video. Green light time predefined in the system for different-different condition of the counted vehicle. According to perfect match condition for detected vehicle found system send the green light counter time.

To decide the different-different green light timer depends upon some parameters. These parameters are: distance and speed. Distance parameter, depends upon the distance which is travelled by the vehicle to cross the intersection area. Distance is different-different for different intersection area. Speed, speed of the waiting vehicles is different from each other and everybody drive their vehicle on different speed. To calculate time of green light signal use a basic formula.

Time $=$ Distance $/$ Speed

Time $=\mathrm{sec}$

Distance $=$ meter

Speed $=$ meter $/ \mathrm{sec}$

In our proposed system we calculate time on the basis of the parameter which is measured by us to calculate signal light time. In this system we calculate and measure parameter from the intersection road at Bhilwara. At this intersection road/area the 100 meter distance travelled by vehicle within $10 \mathrm{sec}$. by average speed. So we take the distance as 100 meter. Speed for the system we take $30 \mathrm{~km} / \mathrm{hr}$. This is calculated on the basis of average speed of all vehicles. According to Indian govt. speed limit for all vehicle is decided. On the basis of their speed we take the average speed.

According to given formula time decided for proposed system:

Time $=\frac{100 \mathrm{~meter}}{30 \mathrm{~km} / \mathrm{hr}}$

Time $=11 \mathrm{sec}$.

A vehicle which is within the 100 meter (including intersection $\mathrm{road} / \mathrm{area}$ ) road is easily able to cross the road within $10 \mathrm{sec}$.

\section{RESULT}

In this section we show the experimental result using data from traffic video. Before applying optical flow estimation on frame the image RGB converted into intensity (gray). Original video and after intensity and median filter shown in fig 1 


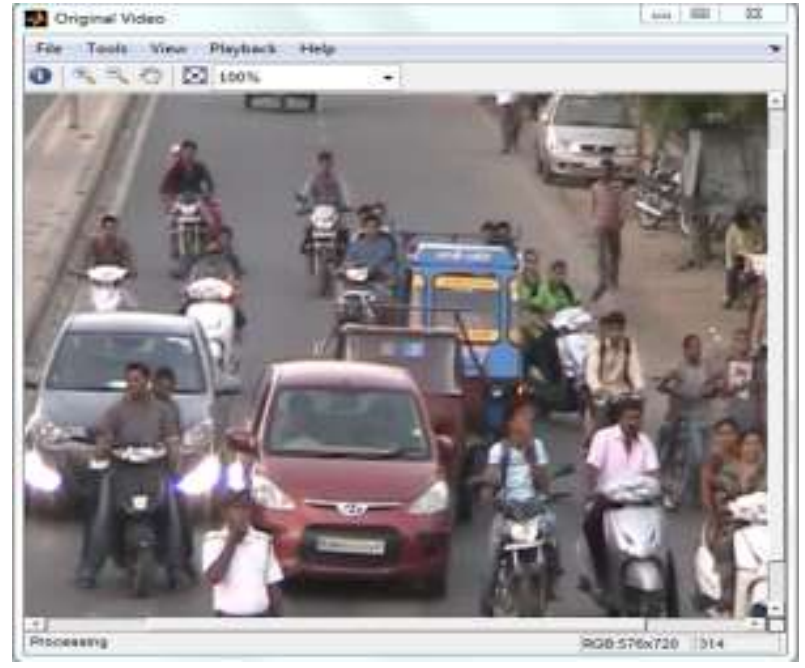

(a)

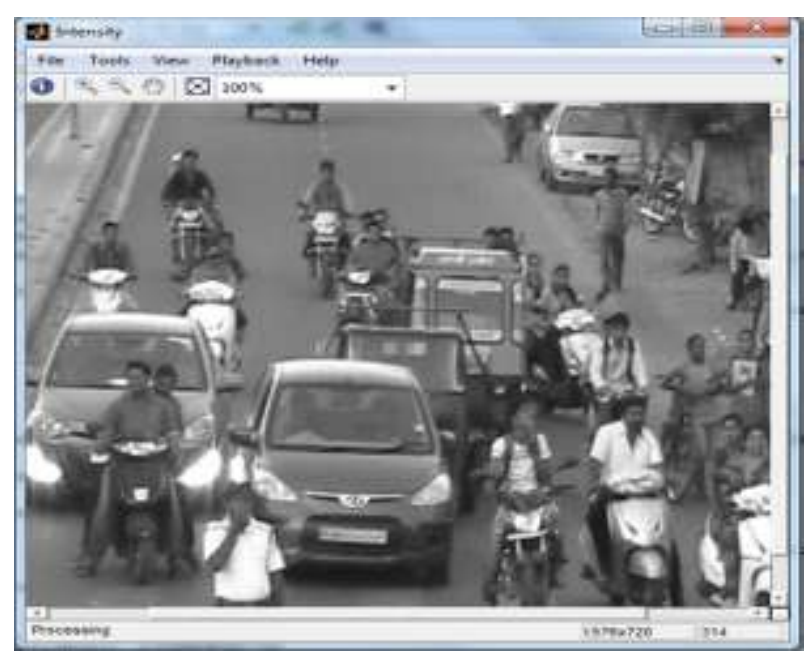

(b)

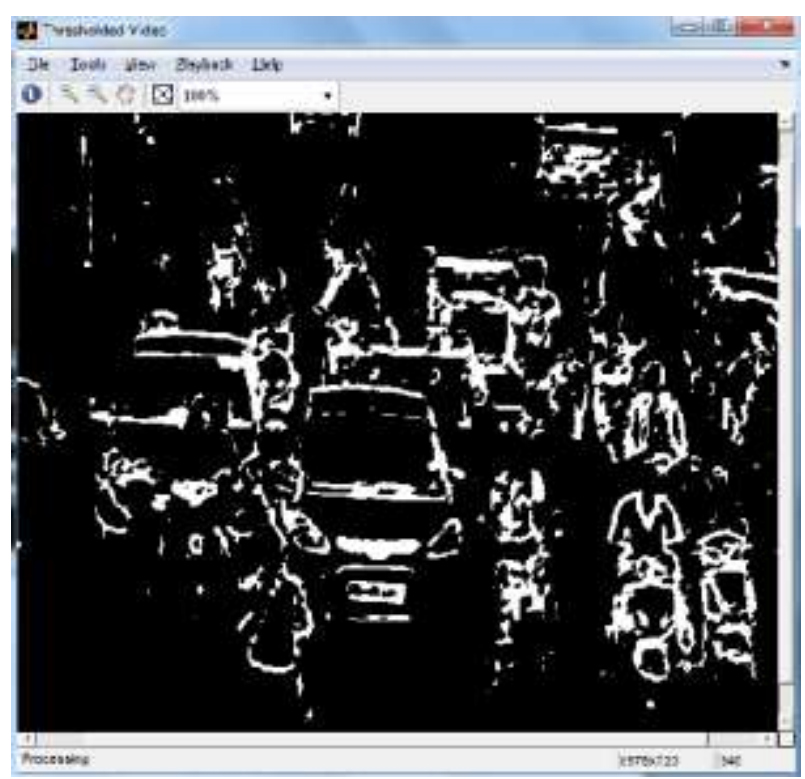

(c)

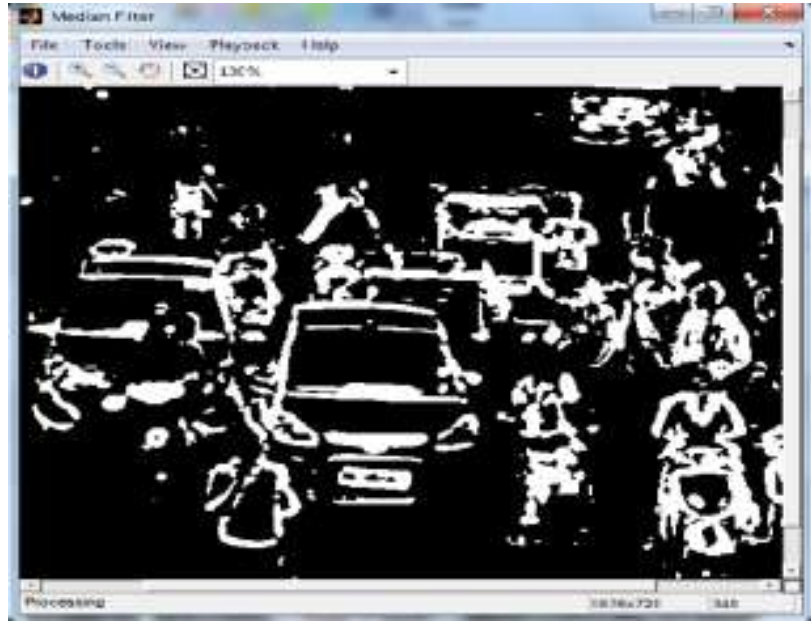

(d)

Figure 1: (a) Original Video (b) Intensity (c) Before filter (d) Median filter

The video analyzed for detecting and counting number of vehicle within the video frame. According to number of vehicle it had shown the time period for green signal light. The system was able to produce results for different scenarios.

Case 1: If Number of vehicles is less than 15 then green signal light timer set to $10 \mathrm{sec}$.

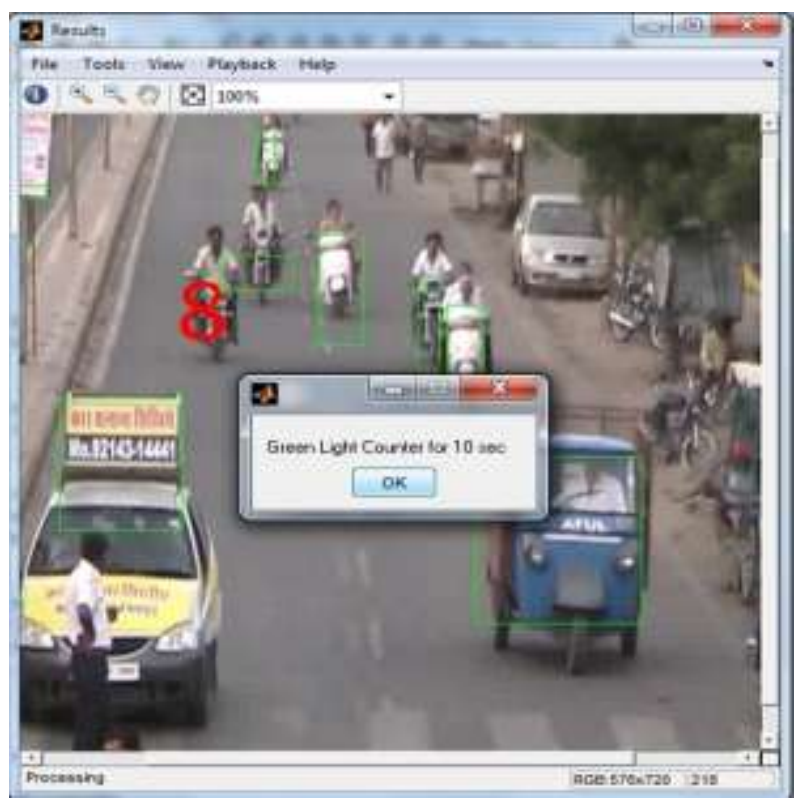

Figure 2: No. of vehicle $=8$, Green light time $=10 \mathrm{sec}$

Case 2: If Number of vehicles is less than 30 then green signal light timer set to $30 \mathrm{sec}$. 


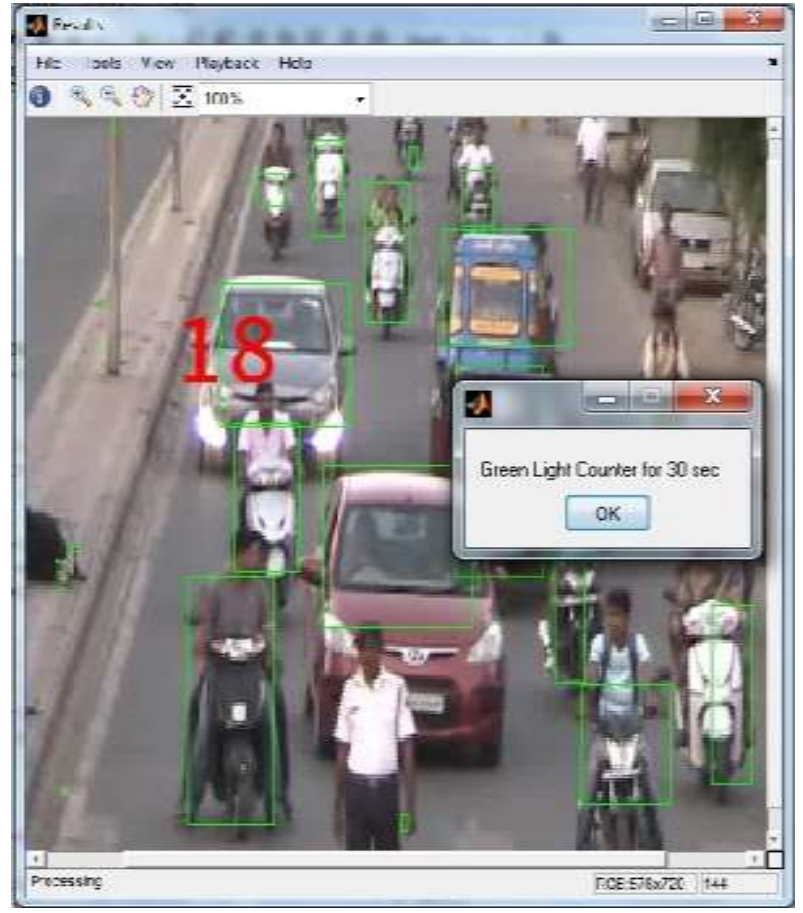

Figure 3: No. of vehicle $=18$, Green light time $=30 \mathrm{sec}$

Case 3: If Number of vehicles is more than 30 then green signal light timer set to $60 \mathrm{sec}$.

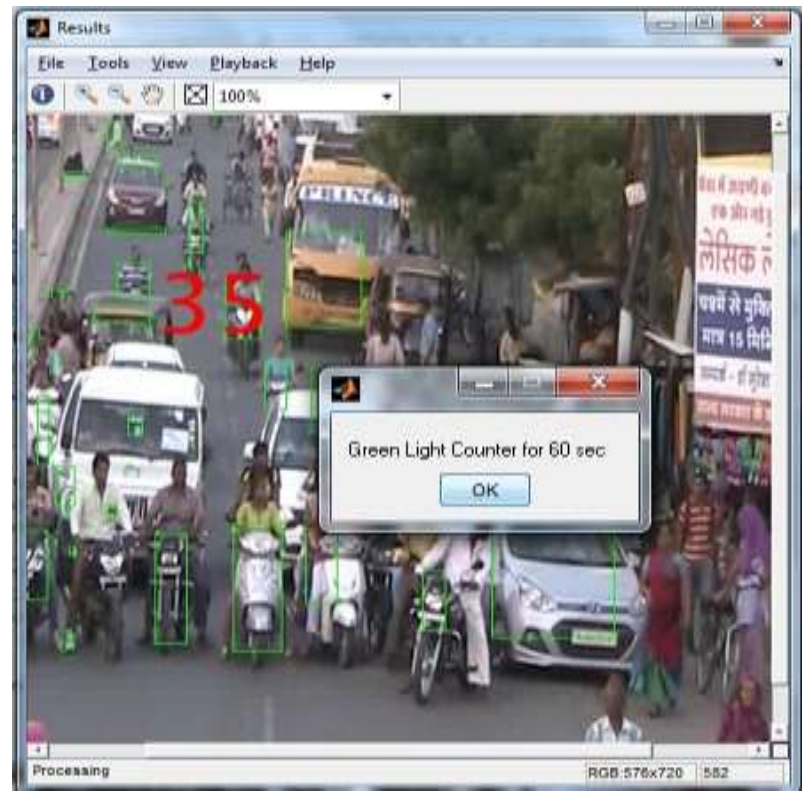

Figure 4: No. of vehicle $=35$ Green light time $=60 \mathrm{sec}$

As experimental result shows green light time counter decided according to number of vehicle detected in the video. When the number of vehicles are increase at signal light green light counter also vary.

\section{RESULT ANALYSIS}

In this chapter we present the test environment and the experimental results analysis of our system.

$\underline{\text { www.ijcat.com }}$

\begin{tabular}{|c|c|c|c|}
\hline $\begin{array}{c}\text { No of } \\
\text { vehicle }\end{array}$ & $\begin{array}{c}\text { Present } \\
\text { System timer } \\
\text { (sec.) }\end{array}$ & $\begin{array}{c}\text { Proposed } \\
\text { System } \\
\text { timer (sec.) }\end{array}$ & $\begin{array}{c}\text { Saved } \\
\text { Time } \\
\text { (sec.) }\end{array}$ \\
\hline 0 & 60 & 5 & 55 \\
\hline$<15$ & 60 & 10 & 50 \\
\hline$<35$ & 60 & 30 & 30 \\
\hline$>35$ & 60 & 60 & 0 \\
\hline
\end{tabular}

Table 1: Result Analysis

In graphical format result analysis can be shown as given below:

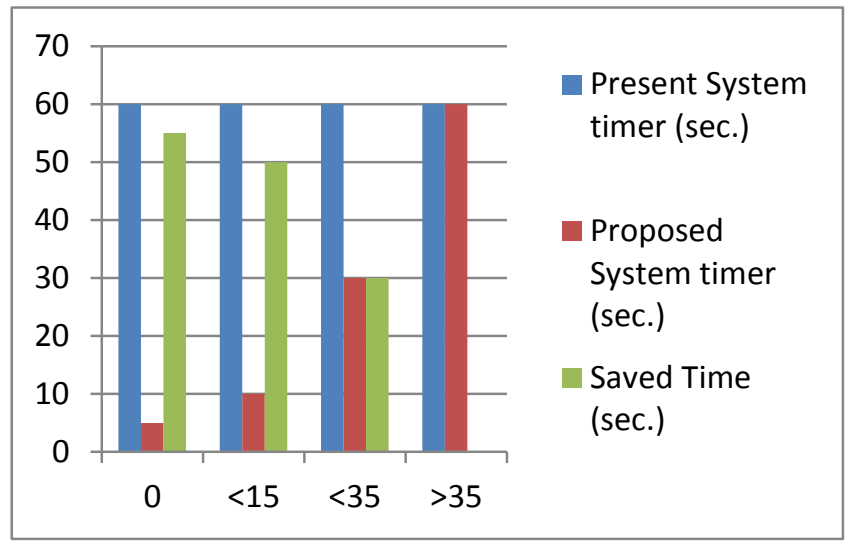

Figure 5: Result Analysis

In present system fuel consumes a lot and it increase air pollution also. According to result analysis it shows we can save a lot of time at traffic signal. Lot of fuel consume by vehicles in waiting time can also be saved. Our proposed system also helps in decrease the fuel consumption by vehicle and decrease the import of fuel from the other countries and saving the foreign currency.

\section{CONCLUSION}

In this thesis we showed that Control Traffic Light Controller using Optical Flow Estimation. We demonstrate on traffic density estimation and manage traffic signal light based on image processing technique and we successfully calculated the traffic and also manage green light counter. It can be reduce the traffic congestion and time wasted by a green light on an empty road. It shows that it can reduce the traffic congestion and avoids the time being wasted by a green light on an empty road. It is also more consistent in detecting vehicle presence because it uses actual traffic images.

\section{FUTURE WORK}

Future work will cover complex testing of the system, and more detailed development of modified algorithms. Also the 
algorithm and identify overlapping objects transparencies during object tracking.

\section{ACKNOWLEDGMENTS}

I would like to thanks, Mr. Shiv Kumar and all CSE department of Mewar University. I also want to thanks my family.

\section{REFERENCES}

[1.] Pranav Maheshwar, Deepanshu Suneja, Praneet Singh, Yogeshwar Mutneja, "Traffic Image Processing System”, Volume 118 - No.2 3, May 2015

[2.] E. Atkociunas, R. Blake, A. Juozapavicius,M. Kazimianec "Image processing in road traffic analysis" Nonlinear Analysis: Modelling and Control, 2005, Vol. 10, No. 4, 315-332.

[3.] Pejman Niksaz, "Automatic traffic estimation using image Processing" International Journal of Signal Processing, Image Processing and Pattern Recognition Vol. 5, No. 4, December, 2012.

[4.] P.D. Kamble, S.P. Untawale and S.B. Sahare, "Application of image processing for traffic queue length” VSRD-MAP, Vol. 2 (5), 2012, 196-205.

[5.] Raad Ahmed Hadi, Ghazali Sulong and Loay Edwar George, "Vehicle Detection and Tracking Techniques: A Concise Review", Vol.5, No.1, February 2014

[6.] Ramesh Navi, Aruna M. G., "Traffic Event Detection using Computer Vision”, Volume 16, Issue 3, Ver. II , May-Jun. 2014.

[7.] Pallavi Choudekar, Sayanti Banerjee, "Real Time Traffic Control using Image Processing”, Vol. 2 No. 1.

[8.] Joonsoo Lee and Alan C. Bovik, "Estimate and Analysis of urban Traffic flow", 2009.

[9.] Pre Kumar.V, Barath. V, Prashanth.K, "Object Counting and density Calculation using MATLAB". 